colours and their proximity and areas.

Colour science is an important but complex subject. Those studying behaviour and ecology are now taking this on board, as several chapters demonstrate. How are receptor signal-tonoise ratios dealt with, and does the Vorobyev and Osorio model best describe most avian colour spaces? What are the advantages and disadvantages of the different approaches to analysing reflectance and illumination spectra, and how do these vary with the different level and type of biological questions posed? How precisely are different cone inputs combined and weighted? What is the taxonomic distribution of spectral sensitivity in birds? How are photonic crystals genetically coded? These are all areas of current intrigue, and quite a few are well covered here.

A sound understanding of colour vision, then, underpins our understanding of bird coloration. This can be a knotty area, with most hard evidence coming from colour-mixing experiments, retinal physiology and sequence data, so contributors specializing in these areas would have been welcome. However, bird coloration is a huge topic and anyone organizing an overview should be heartily congratulated.

Another overview can be found in a special issue of American Naturalist due out this month. Both publications offer much for those studying coloration in other taxa too, because birds provide model systems for investigating animal coloration. Besides, birds are just so exquisitely beautiful.

Andrew T. D. Bennett is at the School of Biological Sciences, University of Bristol, Bristol BS8 1UG, UK.

\title{
A cultural state of mind
}

\section{The Culture of Our Discontent: Beyond the Medical Model of Mental Illness by Meredith F. Small \\ Joseph Henry Press: 2006. 224 pp. \$27.95}

\section{Lewis Wolpert}

In medieval times, madness was regarded as a moral perversion or even satanic possession. There has since been a continuous increase in the use of drugs, from alcohol to antidepressants. The current culture of using pharmaceuticals to treat mental illness concerns the anthropologist Meredith Small. In The Culture of Our Discontent, she examines how such illnesses are viewed in different cultures, and whether, for example, shamans and sorcerers might be able to suggest useful treatments. Much of the book is based on interviews with psychiatrists and anthropologists, and it provides a novel approach to mental illness.

However, Small underestimates how successful talking therapies, such as cognitive therapy, have become. In my view, psychoanalysis, by contrast, is not far removed from witchcraft. One of the problems facing talking therapies is that it is very difficult to do a double-blind clinical trial.

A relatively new approach to psychiatry is based on darwinian evolution. For example, depression is both disabling and common, and has a significant genetic component, so why has it not been selected out? Might depression have a useful function? Could it be a cry for help, or prevent people from pursuing unattainable goals? Most plausible is that sadness is an essential and adaptive human attribute that has become abnormal, much as cancer cells are normal cells that have become malignant. Similar questions relate to schizophrenia. Does it gives someone the advantage of not needing social attention, for example? I don't find this persuasive, however. More relevant, I suggest, is the view that it involves key cognitive processes, such as insight and empathy, that have become abnormal.

Small describes how Harry Harlow used monkeys in the 1950s to try and understand depression. He found that infant monkeys separated from their mothers showed behaviour

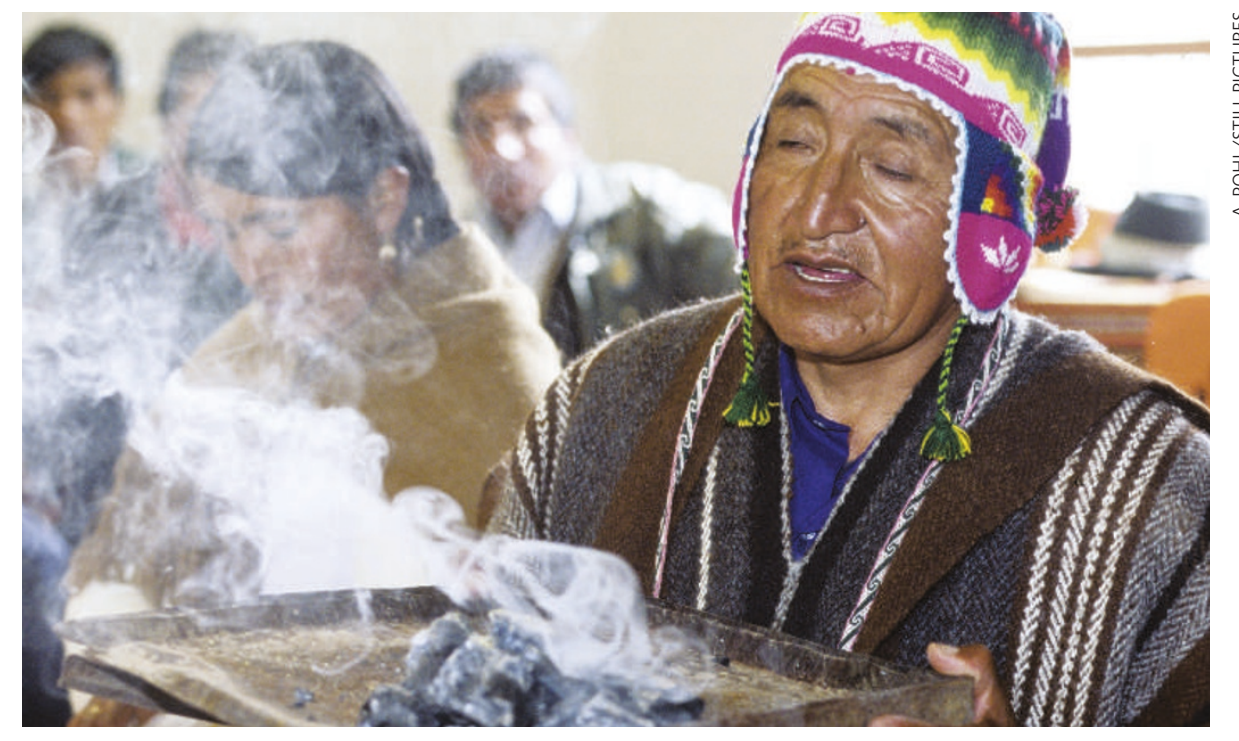

Cultures such as the Aymara in South America have different views of mental illness to the West.

that could be thought of as depression. There was also a strong genetic component. However, they recovered when placed with other young and affectionate monkeys.

Central to the book is the extent to which culture influences the types and incidence of mental illness. Unfortunately, little good evidence is provided in relation to Western illnesses in other cultures, probably because they are not easy to diagnose. There is a widely accepted claim, for example, that the incidence of schizophrenia is $1 \%$ in many different cultures. However, depression is rare in the cold wastes of Iceland, and the number of teenage suicides is growing in Colombia.

Culture, like genes, is handed down and affects behaviour. It also determines what is considered normal. In one Native American community in California, seizures were respected, and in Siberia, many shamans are neurotic. In Botswana, an anthropologist saw a woman suddenly rip off her clothes and run into the bush. Quite slowly, a man went after her and they returned calmly. The locals explained that sometimes people had to do that. In southern China, koro, the male fear of losing the penis, can reach epidemic proportions. By contrast, anorexia is entirely a Western condition and may be related to affluence. Agoraphobia is also Western - there is less need to run for cover in the jungle.

In China there were no acknowledged cases of depression under Mao, but Arthur Kleinman found that patients suffering from neurasthenia - aches and pains - had the characteristics of depression. Such somatization is also common in depressed patients in the West, but this is not explored here.

As Small points out, there are no reliable data on the diagnoses or remedies for different mental illnesses in different cultures, but in the non-Western world there is usually someone to blame, often guided by a witch. Small notes that in Western culture we do not tend to believe in curses, voodoo or magic, but what about religion and much of alternative medicine?

The examples of mental illness in different cultures raise many interesting problems and require further research. We also badly need to improve our understanding of the biology of mental illness, and then the cultural influences may become clearer.

Lewis Wolpert is at University College London,

Gower Street, London WC1E 6BT, UK. 